\title{
Optimización de un nuevo método de preparación de óxidos mixtos tipo perovskita con vacantes de oxígeno
}

\author{
L. MESTRES, M. L. MARTÍNEZ-SARRIÓN, F. J. LEPE
}

Departament de Química Inorgànica, Universitat de Barcelona, Martí i Franquès 1-11, 08028 Barcelona

\begin{abstract}
Se han optimizado las condiciones para la deslitiación de compuestos de fórmula general $\mathrm{La}_{2 / 3-x} \mathrm{Li}_{3 \mathrm{x}} \mathrm{TiO}_{3}$ así como las de la posterior termólisis de compuestos de fórmula general $\mathrm{La}_{2 / 3-x} \mathrm{TiO}_{3-3 \mathrm{x}}(\mathrm{OH})_{3 \times}$. En ambos procesos se mantiene la estructura perovskita cúbica Pm3m. Estudios preliminares de conductividad eléctrica indican que los compuestos $\mathrm{La}_{2 / 3-x} \mathrm{TiO}_{3-3 \mathrm{x} / 2}$ obtenidos mediante química suave a partir de la perovskita de lantano y litio se comportan como semiconductores.
\end{abstract}

Palabras clave: perovskitas, química suave, vacantes de oxígeno

Optimization of a new method to prepare perovskite type mixed oxides with oxygen vacancies

The conditions of both the delithiation of compounds of general formula $\mathrm{La}_{2 / 3-} \mathrm{Li}_{3 \times} \mathrm{TiO}_{3^{\prime}}$ and the thermolisis of compounds of general formula $\mathrm{La}_{2 / 3-\mathrm{x}} \mathrm{TiO}_{3-3 \mathrm{x}}(\mathrm{OH})_{3 \mathrm{x}}$ have been optimised. In both processes the cubic perovskite structure Pm3m is maintained. Preliminary studies of the electrical conductivity show that the $\mathrm{La}_{2 / 3-x} \mathrm{TiO}_{3-3 \times 2 / 2}$ compounds obtained through soft chemistry from lanthanum and lithium perovskite, behave as semiconductors.

Key words: perovskites, soft chemistry, oxygen vacancies

\section{INTRODUCCIÓN}

Las perovskitas ideales de fórmula $\mathrm{ABO}_{3}$ muestran una simetría Pm3m. Dichas perovskitas presentan un gran número de propiedades, tales como conductividad iónica (1), superconductividad (2), magnetorresistencia (3) y ferroelectricidad (4). Los sólidos que presentan estructuras relacionadas con las perovskitas se sintetizan empleando altas temperaturas, o bien mediante métodos de química suave utilizando temperaturas más bajas. Otro de los métodos utilizados es el intercambio iónico, mediante el cuál pueden prepararse fases termodinámicamente inestables. Suelen obtenerse materiales de estructura semejante a la del compuesto de partida, por lo que es importante usar los precursores adecuados para obtener el producto deseado. Mediante este procedimiento se ha preparado una perovskita cúbica $\mathrm{KNbO}_{3}$ a partir de la perovskita en capas $\mathrm{K}_{2} \mathrm{NbO}_{3} \mathrm{~F}$ (5).

Los titanatos de litio y lantano de la serie $\mathrm{La}_{2 / 3-x} \mathrm{Li}_{3 \mathrm{x}} \mathrm{TiO}_{3}$ han sido objeto de numerosos trabajos por el interés de su estructura (6) y por la elevada conductividad iónica de litio que presentan $(1,7)$. Es bien conocido que los óxidos que contienen iones alcalinos pueden intercambiar dichos iones por protones, siendo utilizados como precursores en la síntesis de óxidos metaestables. Un ejemplo sería el intercambio de iones sodio por protones en el óxido $\mathrm{Na}_{2} \mathrm{Nd}_{2} \mathrm{Ti}_{3} \mathrm{O}_{10}$ para dar $\mathrm{H}_{2} \mathrm{Nd}_{2} \mathrm{Ti}_{3} \mathrm{O}_{10^{\prime}}$ el cual por deshidratación conduce al óxido metaestable $\mathrm{Nd}_{2 / 3} \mathrm{TiO}_{3}(8)$.

Se pretende en este trabajo preparar compuestos con estructura perovskita de fórmula general $\mathrm{La}_{2 / 3-\mathrm{x}} \mathrm{TiO}_{3-3 \times / 2}$ con vacantes aniónicas. La presencia de vacantes de oxígeno en estos óxidos permitirá su utilización como electrolitos en celdas de combustible de óxidos sólidos (SOFCs), o bien como sensores de oxígeno. Su síntesis por el método cerámico presenta problemas ya que se requiere una atmósfera altamente reductora para provocar la aparición de dichas vacantes. Para solventar esta dificultad se propone la síntesis de los compuestos del sistema $\mathrm{La}_{2 / 3-x} \mathrm{TiO}_{3-3 x / 2}$ a partir de compuestos de fórmula general $\mathrm{La}_{2 / 3-x} \mathrm{Li}_{3 \mathrm{x}} \mathrm{TiO}_{3}$ mediante la extracción de litio en condiciones suaves.

La síntesis de fases metaestables por métodos de química suave tiene lugar siguiendo el esquema que se presenta a continuación (Figura 1).

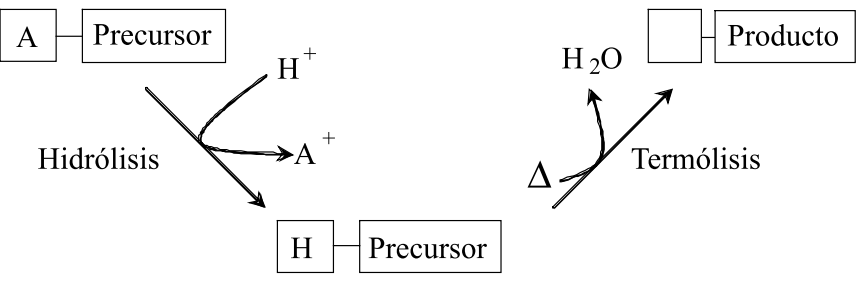

Figura 1. Esquema de la síntesis de fases metaestables por métodos de química suave

\section{PARTE EXPERIMENTAL Y RESULTADOS}

\subsection{Síntesis cerámica del precursor}

La síntesis de los óxidos de fórmula general $\mathrm{La}_{2 / 3-x} \mathrm{Li}_{3 \mathrm{x}} \mathrm{TiO}_{3}$ se llevó a cabo a partir de $\mathrm{TiO}_{2}$ (Aldrich 99,9\%), $\mathrm{La}_{2} \mathrm{O}_{3}$ (Fluka 99,98\%), ambos calentados previamente a $900^{\circ} \mathrm{C}$ durante 12 horas para deshidratarlos, y $\mathrm{Li}_{2} \mathrm{CO}_{3}$ (Aldrich 99\%). Se pesaron las cantidades estequiométricas necesarias añadiendo un exceso del $10 \%$ de $\mathrm{Li}_{2} \mathrm{CO}_{3}$ para paliar las pérdidas del litio durante los tratamientos térmicos. Las mezclas de los reactivos se homogeneizaron en acetona en un mortero de ágata, y se 
colocaron en una estufa a $120^{\circ} \mathrm{C}$ para evaporar el disolvente. Una vez secas las muestras se colocaron en navecillas de platino y se calentaron en una mufla a $650^{\circ} \mathrm{C}$ durante tres horas para conseguir su completa descarbonatación. El polvo obtenido se trituró y prensó en forma de pastillas de aproximadamente $1 \mathrm{~g}$, se colocaron en navecillas de platino y se recubrieron con polvo de la misma composición para evitar pérdidas de litio por volatilización, y se taparon con navecillas de platino. Las muestras fueron sometidas a los siguientes tratamientos térmicos: 15 horas a $1100^{\circ} \mathrm{C}, 15$ horas a $1200^{\circ} \mathrm{C}$ y $15 \mathrm{~h}$ a $1250^{\circ} \mathrm{C}$, con triturado, homogeneizado y prensado entre cada tratamiento. Al finalizar el último tratamiento se lleva a cabo un quenching a temperatura ambiente.

\subsubsection{CARACTERIZACIÓN DEL PRECURSOR}

Para hacer la difracción de rayos $\mathrm{X}$ sobre polvo cristalino se ha utilizado un difractómetro de geometría Bragg-Brentano $\theta / 2 \theta$ Siemens D-500, con monocromador secundario de grafito. La radiación utilizada ha sido la Ko del cobre a $40 \mathrm{kV}$ y $30 \mathrm{~mA}$.

Las especies se ajustan a una fase cúbica Pm3m. En la Figura 2 se muestra como ejemplo, el diagrama de difracción para la composición $\mathrm{La}_{0,57(1)} \mathrm{Li}_{0,29(1)} \mathrm{TiO}_{3}$ ajustado mediante el programa Fullprof (9). En la Tabla I se muestran se los parámetros de celda de los diferentes óxidos preparados.

TABLA I. COMPOSICIONES Y PARÁMETROS DE LOS ÓXIDOS PRECURSORES

\begin{tabular}{|c|c|c|c|}
\hline $\begin{array}{c}\text { Composición } \\
\text { teórica }\end{array}$ & $\begin{array}{c}\text { Composición } \\
\text { experimental }\end{array}$ & $\mathrm{a}(\AA)$ & $\chi^{2}$ \\
\hline $\mathrm{La}_{0,60} \mathrm{Li}_{0,21} \mathrm{TiO}_{3}$ & $\mathrm{La}_{0,60(1)} \mathrm{Li}_{0,21(1)} \mathrm{TiO}_{3}$ & $3,8765(7)$ & 5,1 \\
\hline $\mathrm{La}_{0,58} \mathrm{Li}_{0,25} \mathrm{TiO}_{3}$ & $\mathrm{La}_{0,58(1)} \mathrm{Li}_{0,25(1)} \mathrm{TiO}_{3}$ & $3,8752(7)$ & 5,2 \\
\hline $\mathrm{La}_{0,57} \mathrm{Li}_{0,29} \mathrm{TiO}_{3}$ & $\mathrm{La}_{0,57(1)} \mathrm{Li}_{0,29(1)} \mathrm{TiO}_{3}$ & $3,8742(7)$ & 5,2 \\
\hline $\mathrm{La}_{0,55} \mathrm{Li}_{0,34} \mathrm{TiO}_{3}$ & $\mathrm{La}_{0,55(1)} \mathrm{Li}_{0,34(1)} \mathrm{TiO}_{3}$ & $3,8728(7)$ & 4,0 \\
\hline $\mathrm{La}_{0,54} \mathrm{Li}_{0,39} \mathrm{TiO}_{3}$ & $\mathrm{La}_{0,54(1)} \mathrm{Li}_{0,39(1)} \mathrm{TiO}_{3}$ & $3,8708(7)$ & 5,0 \\
\hline
\end{tabular}

Las composiciones de los diferentes óxidos sintetizados (Tabla I) se obtuvieron a partir de los datos de espectroscopia de emisión por plasma acoplado inductivamente (AES-ICP), realizada en un espectrómetro de emisión Perkin Elmer modelo Optima 3200 RL.

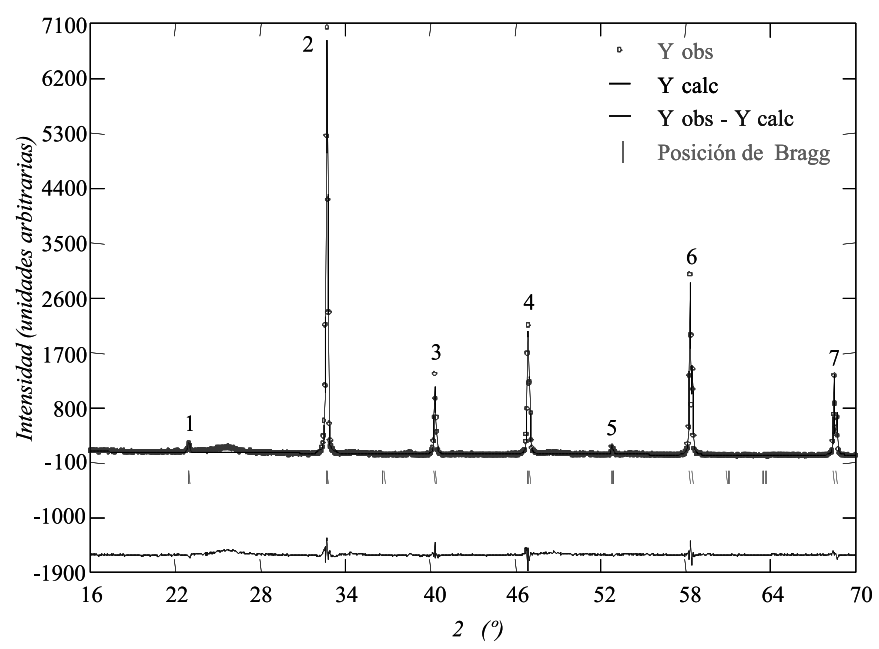

Figura 2. Diagrama de difracción de rayos X para la composición $\mathrm{La}_{0,57(1)} \mathrm{Li}_{0,29(1)} \mathrm{TiO}_{3}$

\subsection{Procedimiento experimental del proceso de deslitiación}

En un balón tipo schlenk de $100 \mathrm{~mL}$ se introducen $0,500 \mathrm{~g}$ de precursor finamente triturado y $50 \mathrm{~mL}$ de ácido nítrico $2 \mathrm{M}$, se termostatiza la muestra a la temperatura deseada y se deja reaccionar durante el tiempo requerido con agitación constante para favorecer el proceso de la difusión de los iones. Finalmente se seca la muestra en una estufa a $120^{\circ} \mathrm{C}$ durante toda la noche y se guarda en un desecadaor de gel de sílice. Las condiciones para este proceso que aparecen en la bibliografía (10) son: tiempo 5 dias, temperatura $60^{\circ} \mathrm{C}$, concentración del ácido nítrico $2 \mathrm{M}$. Los resultados obtenidos con estas condiciones no fueron satisfactorios ya que la deslitiación no fue completa, por lo que se establecieron unas nuevas condiciones. Las condiciones establecidas en este trabajo con las que se consigue que la deslitiación sea total son las que damos a continuación. Tiempo de reacción: siete días, temperatura $90^{\circ} \mathrm{C}$, concentración de $\mathrm{HNO}_{3} 2 \mathrm{M}$, y por último, la especie a deslitiar puede ser en forma de polvo cristalino o pastilla previamente sinterizada a $1250^{\circ} \mathrm{C}$ durante una hora.

\subsubsection{CARACTERIZACIÓN DE LOS COMPUESTOS DESLITIADOS}

La Tabla II muestra las composiciones de las especies deslitiadas obtenidas a partir de los compuestos de la Tabla I. Dichas composiciones se han obtenido mediante análisis de ICP. Las muestras deslitiadas se estudiaron mediante difracción de rayos $\mathrm{X}$. Los compuestos mantienen la simetría Pm3m con pequeñas variaciones en los parámetros de celda

TABLA II. COMPOSICIONES DE LOS ÓXIDOS PRECURSORES, DESLITIADOS Y TERMOLIZADOS

\begin{tabular}{|c|c|c|c|}
\hline \multicolumn{3}{|c|}{ Composición de los óxidos } & \multirow{2}{*}{$\mathrm{a}(\AA)$} \\
\hline precursores & deslitiados & termolizados & \\
\hline $\mathrm{La}_{0,60(1)} \mathrm{Li}_{0,21(1)} \mathrm{TiO}_{3}$ & $\mathrm{La}_{0,6011} \mathrm{TiO}_{2,79}(\mathrm{OH})_{0,21}$ & $\mathrm{La}_{0,60(1)} \mathrm{TiO}_{2,90}$ & 3,8838(8) \\
\hline $\mathrm{La}_{0,58(1)} \mathrm{Li}_{0,25(1)} \mathrm{TiO}_{3}$ & $\mathrm{La}_{0,58(1)} \mathrm{TiO}_{2,75}(\mathrm{OH})_{0,25}$ & $\mathrm{La}_{0,58(1)} \mathrm{TiO}_{2,87}$ & $3,8826(8)$ \\
\hline $\mathrm{La}_{0,57(1)} \mathrm{Li}_{0,29(1)} \mathrm{TiO}_{3}$ & $\mathrm{La}_{0,57(1)} \mathrm{TiO}_{2,71}(\mathrm{OH})_{0,29}$ & $\mathrm{La}_{0,57(1)} \mathrm{TiO}_{2,86}$ & $3,8814(8)$ \\
\hline $\mathrm{La}_{0,55(1)} \mathrm{Li}_{0,34(1)} \mathrm{TiO}_{3}$ & $\mathrm{La}_{0,55(1)} \mathrm{TiO}_{2,66}(\mathrm{OH})_{0,34}$ & $\mathrm{La}_{0,55(1)} \mathrm{TiO}_{2,83}$ & $3,8803(8)$ \\
\hline $\mathrm{La}_{0,54(1)} \mathrm{Li}_{0,39(1)} \mathrm{TiO}_{3}$ & $\mathrm{La}_{0,5411} \mathrm{TiO}_{2,61}(\mathrm{OH})_{0,39}$ & $\mathrm{La}_{0,54(1)} \mathrm{TiO}_{2,81}$ & $3,8784(8)$ \\
\hline
\end{tabular}

\subsection{Termólisis de los compuestos deslitiados}

Este paso es crítico para la obtención del producto final con la estructura deseada y en la forma adecuada para el estudio de sus propiedades eléctricas. En la termólisis se ha utilizado la muestra deslitiada en forma de pastilla, ya que las medidas eléctricas se harán sobre pastillas. Hay que ajustar los siguientes parámetros: velocidad de calentamiento de la muestra, temperatura y tiempo de tratamiento y, atmósfera a utilizar. La atmósfera que se utilizó fue nitrógeno ya que con ello se evita la posible descomposición de las muestras en otros óxidos de lantano y titanio. La sinterización se llevó a cabo teniendo en cuenta los datos de la bibliografía (10) $\left(800^{\circ} \mathrm{C}\right.$, durante 5 minutos y $800^{\circ} \mathrm{C}$ durante 12 horas). Viendo que los resultados no eran satisfactorios, y con los datos obtenidos mediante análisis térmico diferencial se realizaron diversos tratamientos térmicos hasta establecer las condiciones óptimas que son las siguientes: $950^{\circ} \mathrm{C}$, durante 60 horas, y una velocidad de calentamiento de una hora por minuto. 


\subsubsection{CARACTERIZACIÓN DE LOS COMPUESTOS DESPUÉS DE LA TERMÓLISIS}

En la Tabla II se dan los resultados de ICP de las muestras que se han sometido al proceso de termólisis. En la Figura 3 se da el diagrama de difracción de la muestra $\mathrm{La}_{0,57(1)} \mathrm{TiO}_{2,86}$. Se trata de nuevo de una fase cúbica de simetría Pm3m. La Tabla II muestra el valor del parámetro $a$ para cada composición después de la termólisis.

\subsection{Caracterización mediante resonancia paramagnética elec- trónica (EPR)}

Los espectros de EPR se registraron en la banda X $(9,4 \mathrm{GHz})$ con un espectrómetro Brucker ESP-300E a temperatura ambiente y a 77K.

En el espectro EPR de la composición $\mathrm{La}_{0,57(1)} \mathrm{TiO}_{2,86}$ a $77 \mathrm{~K}$ se observa una señal centrada en $\mathrm{g}=2,000$, con una anchura menor de $10 \mathrm{G}$, similar a la de un radical orgánico, por lo que difícilmente podría tratarse de la señal correspondiente al $\mathrm{Ti}^{3+}$, que sería bastante más ancha. Este hecho está de acuerdo con el color blanco de la muestra. Dado que el compuesto objeto de estudio presenta gran número de vacantes aniónicas, esta señal se ha relacionado con la presencia de centros de color en la estructura. Estos centros de color se pueden formar cuando algunos electrones quedan atrapados en las vacantes de oxígeno. En nuestro caso, el tratamiento final de larga duración y a temperatura bastante elevada podría favorecer la formación de estos centros, ya que creamos vacantes de oxígeno, y algunos electrones podrían retenerse en dichas vacantes. En la bibliografía aparecen diversos ejemplos de formación de centros de color, como es el caso del cuarzo natural, que presenta una señal de resonancia paramagnética electrónica del mismo tipo que la observada para esta perovskita.

\subsection{Medidas eléctricas preliminares}

Las medidas eléctricas se llevaron a cabo mediante espectroscopia de impedancias (IS) en un analizador de frecuencias HP 4192 en un intervalo de frecuencias de $5 \mathrm{~Hz}$ a $13 \mathrm{MHz}$ con temperaturas y atmósferas diferentes. Los valores de los diferentes componentes de los circuitos se obtuvieron mediante el ajuste de los datos con el programa Zview 2 (11).

Las muestras se prepararon en forma de pastillas de $6 \mathrm{~mm}$ de diámetro y $2 \mathrm{~mm}$ de grosor. Las muestras previamente sinterizadas que se han medido son $\mathrm{La}_{0,57(1)} \mathrm{TiO}_{2,86}$ y el óxido precursor $\mathrm{La}_{0,57(1)} \mathrm{Li}_{0,29(1)} \mathrm{TiO}_{3}$. Las caras de las pastillas se recubrieron con pasta de oro y se colocaron dos hilos de platino, se secaron en la estufa a $120^{\circ} \mathrm{C}$ durante 10 minutos y se sometieron a calentamiento desde temperatura ambiente hasta $700^{\circ} \mathrm{C}$ en un tiempo de seis horas. Para la muestra deslitiada se empleó atmósfera de nitrógeno.

En la Figura 4 se representa la impedancia real $\left(Z_{R}\right)$ frente a la impedancia imaginaria $\left(\mathrm{Z}_{\mathrm{I}}\right)$ a $46^{\circ} \mathrm{C}$ y el circuito equivalente para el óxido precursor $\mathrm{La}_{0,57(1)} \mathrm{Li}_{0,29(1)} \mathrm{TiO}_{3}$. A altas frecuencias se observa el primer semicírculo que corresponde a la respuesta del grano tiene una capacidad de $\quad 10^{-11} \mathrm{~F} / \mathrm{cm}$ y una conductividad asociada del orden de $10^{-3}$ $\mathrm{S} / \mathrm{cm}$. A frecuencias medias puede apreciarse parte de un semicírculo de radio mayor que el anterior, con una capacidad de $10^{-9} \mathrm{~F} / \mathrm{cm}$ y una conductividad del orden de $10^{-4} \mathrm{~S} / \mathrm{cm}$, que se asocia a la frontera de grano. A bajas frecuencias se observa un spike que se atribuye a los electrodos utilizados que son bloqueantes al paso de los portadores de carga, en este caso los iones litio. Para poder explicar el comportamiento tipo no-Debye asociado al grano y a la frontera de grano se han conectado en paralelo a los circuitos RC correspondientes, elementos

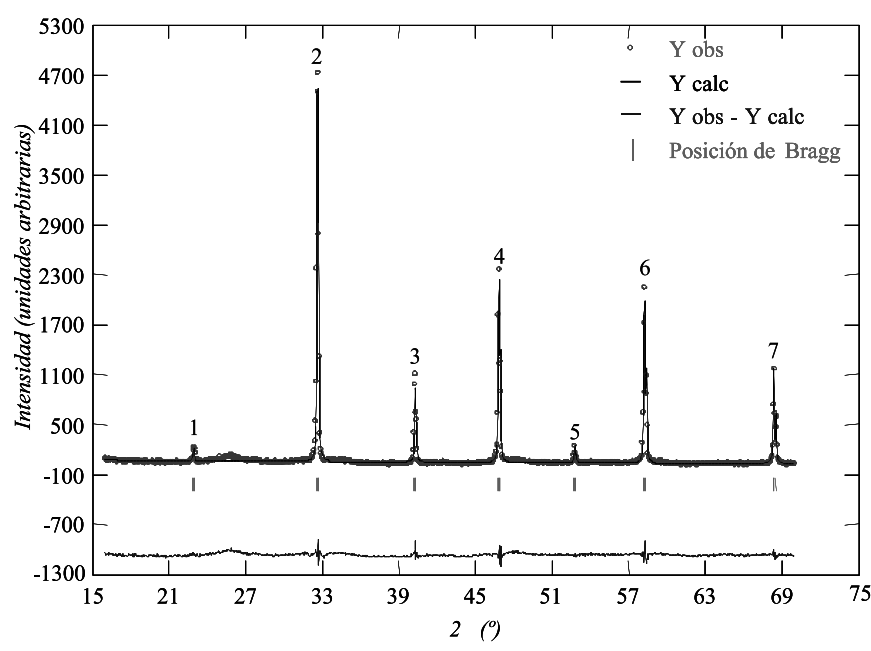

Figura 3. Diagrama de difracción de rayos $\mathrm{X}$ para la composición $\mathrm{La}_{0,57(1)} \mathrm{TiO}_{2,86}$

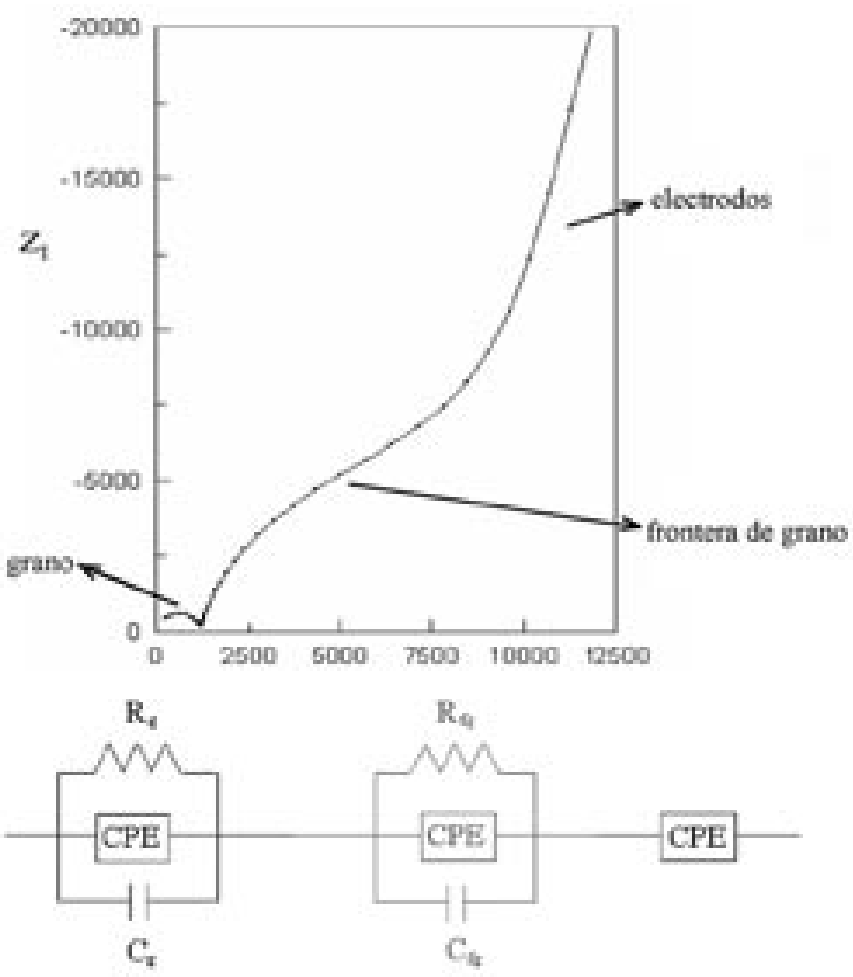

Figura 4. $\mathrm{Z}_{\mathrm{I}}$ vs $\mathrm{Z}_{\mathrm{R}}$ del óxido $\mathrm{La}_{0,57(1)} \mathrm{Li}_{0,29(1)} \mathrm{TiO}_{3}$ a $46^{\circ} \mathrm{C}$ y circuito equivalente

de fase constante. También se ha utilizado un elemento de fase constante conectado en serie con los circuitos RC para simular el spike observado a bajas frecuencias. A partir de los valores de la conductividad de grano se representa $\log (\sigma \mathrm{T})$ en función de 1000 / T en la Figura 4 . El valor de la energía de activación calculado en la gráfica de Arrhenius es de $0,35 \mathrm{eV}$.

Las medidas eléctricas realizadas sobre la especie deslitiada $\mathrm{La}_{0,57(1)} \mathrm{TiO}_{2,86}$ aparecen en las Figuras 6a) y 6b). A temperatura ambiente la representación es una línea vertical, centrada en cero, que se asocia a la respuesta de un material dieléctrico que se comporta como un capacitor, con una capacidad de $10^{-11} \mathrm{~F} / \mathrm{cm}$. Al aumentar la temperatura, 


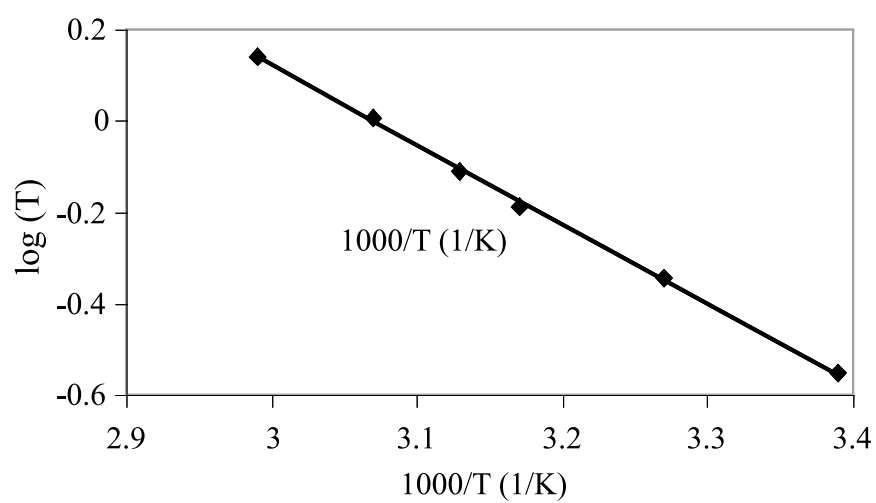

Figura 5. Gráfica de Arrhenius del óxido $\mathrm{La}_{0,57(1)} \mathrm{Li}_{0,29(1)} \mathrm{TiO}_{3}$
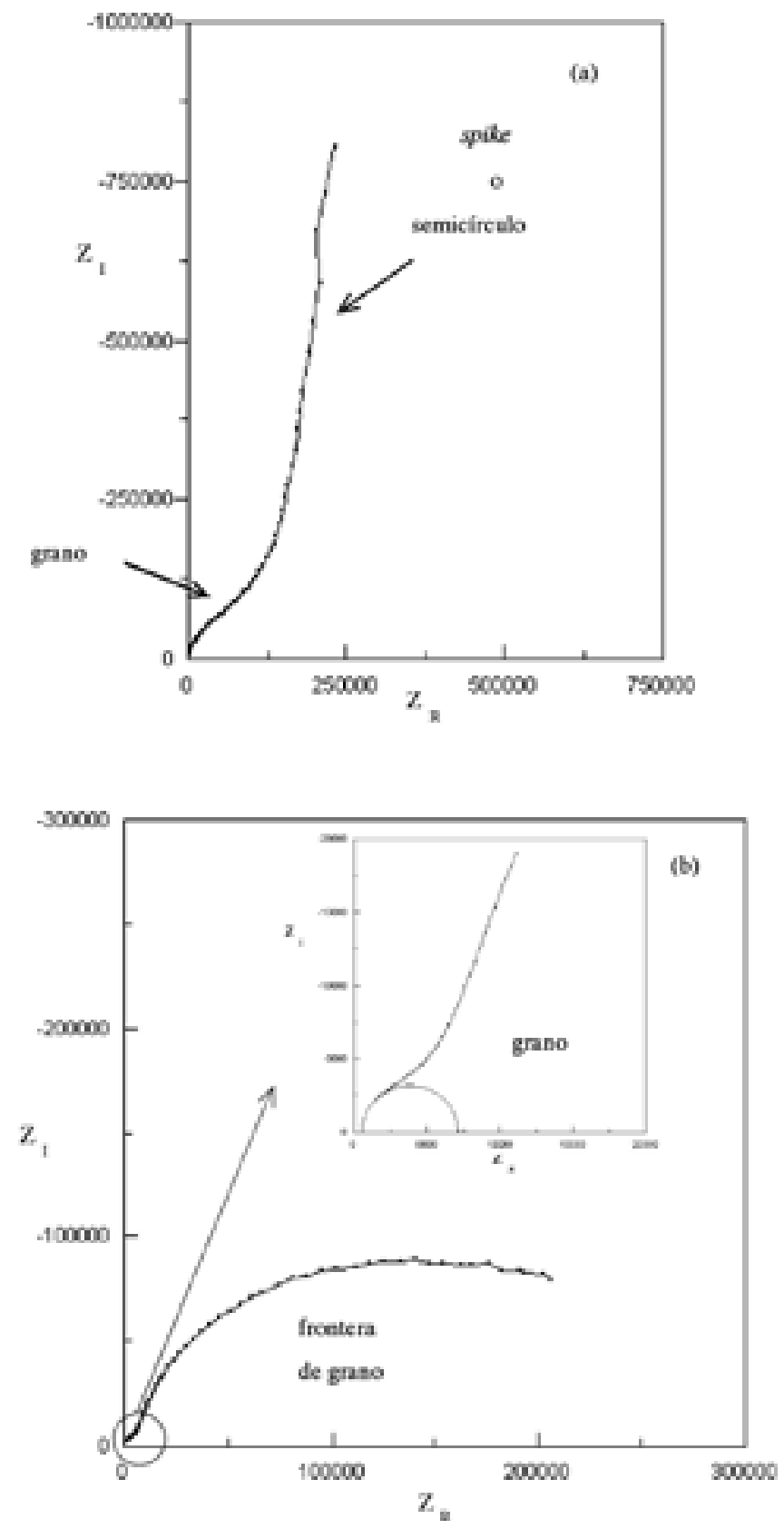

Figura 6. $\mathrm{Z}_{\mathrm{I}}$ vs $\mathrm{Z}_{\mathrm{R}}$ del óxido $\mathrm{La}_{0,57(1)} \mathrm{TiO}_{2,86}$ a) a $231^{\circ} \mathrm{C}$ y b) a $508^{\circ} \mathrm{C}$
Figura 6a), se inicia un semicírculo en la zona de altas frecuencias cuya capacidad es del orden de $10^{-11} \mathrm{~F} / \mathrm{cm}$ lo cual indicaría que se trata de respuesta del grano, y la zona de bajas frecuencias podría corresponder a un spike o a un semicírculo de elevada resistencia. Para aclarar esto se hicieron medidas a temperaturas superiores, concretamente la Figura $6 \mathrm{~b}$ ) muestra la que corresponde a $508^{\circ} \mathrm{C}$. Lo que aparecía como un spike a $231^{\circ} \mathrm{C}$ se ha cerrado en forma de semicírculo con una capacidad asociada de $10^{-10} \mathrm{~F} / \mathrm{cm}$, que se puede relacionar con la frontera de grano. La temperatura se incrementó hasta $607^{\circ} \mathrm{C}$, obteniéndose una representación como la 6 b) con descenso de los valores de las resistencias asociadas a los semicírculos. El ajuste de las medidas mediante circuitos equivalentes es complejo debido a las variaciones de la respuesta con la temperatura, sin embargo en las Figuras 7a) y 7b) se dan los circuitos equivalentes para bajas y altas temperaturas respectivamente. En ambos casos se han empleado elementos de fase constante para mejorar el ajuste. A partir de los valores de la conductividad de grano y mediante una gráfica de Arrhenius, Figura 8, se puede calcular la energía de activación que es aproximadamente de 0,33 eV. Para las muestras deslitiadas y termolizadas, por tanto, la conductividad parece tener su origen en los centros de color como se comentó anteriormente. Así los portadores de carga serían los electrones, y la ausencia de un spike en las representaciones de $Z_{I}$ vs $Z_{R}$ estaría justificada, ya que los electrodos no serían bloqueantes al paso de los electrones. Igualmente, sería posible la presencia de un nuevo elemento en la zona de bajas frecuencias que correspondería a la interfase muestra-electrodo. Esta posibilidad se comenta en la bibliografía, aunque los diagramas Cole-Cole son diferentes (10).
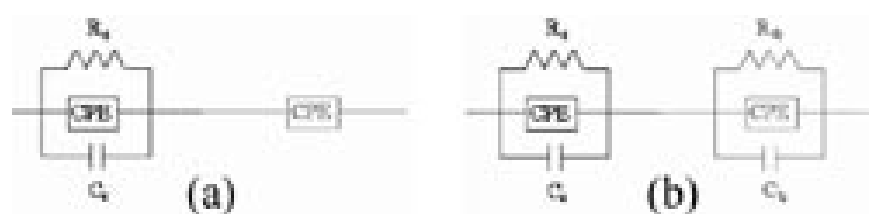

Figura 7. Circuitos equivalentes de la composición $\mathrm{La}_{0,57(1)} \mathrm{TiO}_{2,86}$ a) hasta $318^{\circ} \mathrm{C}, \mathrm{b}$ ) hasta $607^{\circ} \mathrm{C}$

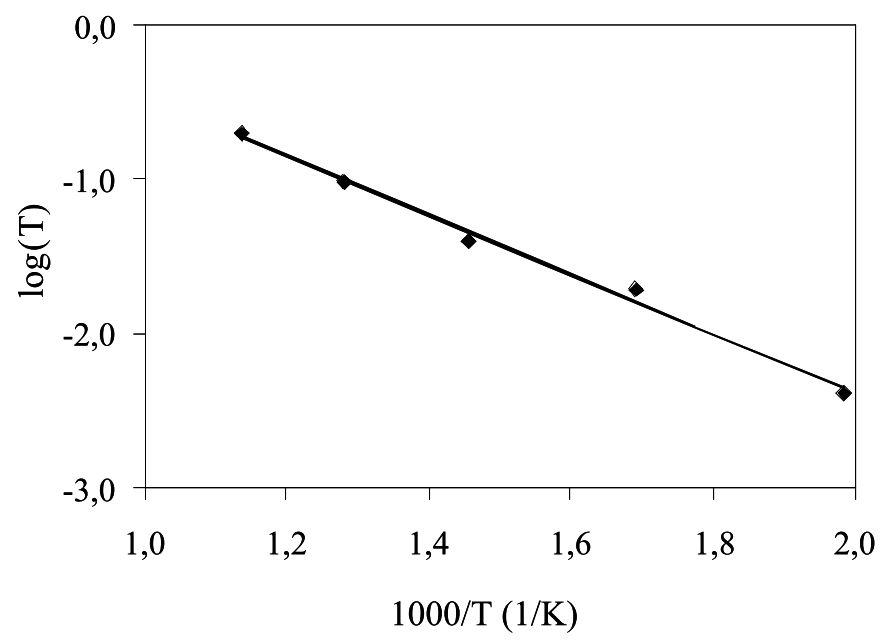

Figura 8. Gráfica de Arrhenius del óxido $\mathrm{La}_{0,57(1)} \mathrm{TiO}_{2,86}$ 


\section{CONCLUSIONES}

Se han optimizado las condiciones experimentales para obtener mediante química suave perovskitas de fórmula general $\mathrm{La}_{2 / 3-x} \mathrm{TiO}_{3-3 \times / 2}$ a partir de $\mathrm{La}_{2 / 3-x} \mathrm{Li}_{3 \mathrm{x}} \mathrm{TiO}_{3}$. Este método se aplicará a la obtención de compuestos con vacantes de oxígeno y se procederá al estudio de las propiedades eléctricas en atmósfera de oxígeno para comprobar su posible aplicación como electrolitos en celdas de combustible de óxidos sólidos o bien como sensores de oxígeno.

\section{AGRADECIMIENTOS}

Este trabajo ha sido parcialmente financiado por el Ministerio de Ciencia y Tecnología, Proyecto BQU2002-00619, y la Generalitat de Catalunya, Proyecto 2001SGR00052.

\section{BIBLIOGRAFÍA}

1. Y. Inaguma, C. Liquan, M. Itoh, T. Nakamura, T. Uchida, H. Ikuta, M. Wakihara, "High conductivity in lithium lanthanum titanate", Solid State Común., 86 689-693 (1993)

2. H. Fukuoka, T Isami, S. Yamanaka, "Superconductivity of alkali metal inter- calated niobate with a layered perovskite structure", Chem. Lett., (8) 703-704 (1997)

3. Y. Moritomo, A. Asamitsu, H. Kuwahara, Y. Tokura, “Giant magnetoresistance of manganese oxides with a layered perovskite structure", Nature, 380 141-144 (1996)

4. W. A. Schulze, J.V. Biggers, L.E. Cross, "Aging of dielectric dispersion in PLZT relaxor ceramics", J. Am. Ceram. Soc., 61 46-49 (1996)

5. K. Toda, S. Tokuoka, K. Uematsu, M. Sato, “Room temperature synthesis and characterization of perovskite compounds", Solid State Ionics, 154-155, 393-398 (2002)

6. A. Várez, F. García-Alvarado, E. Morán, M.A. Alario-Franco, “Microestructural Study of $\mathrm{La}_{05} \mathrm{Li}_{05} \mathrm{TiO}_{3}$ ", J. Solid State Chem., 118, 78-83 (1995)

7. J. Emery, J.Y.Buzare, O. Bohnké, J.L. Fourquet, "Lithium-7 NMR and ionic conductivity studies of lanthanum lithium titanate electrolytes", Solid State Ionics, 99, 41-51 (1997)

8. R. Marchand, L. Brohan, M. Tournoux, " $\mathrm{TiO}_{2}(\mathrm{~B}) \mathrm{A}$ new form of titanium dioxide and the potassium octatitanate $\mathrm{K}_{2} \mathrm{Ti}_{8} \mathrm{O}_{17}$ ", Mater. Res. Bull., 15(8), 1129-1133 (1980)

9. J. Rodriguez Carvajal, Fullprof: A program for Rietvel refinement and pattern machine analysis, revised version, 1994.

10. N.S.P. Bhuvanesh, O. Bohnké, H. Duroy, M.P. Crosnier-Lopez, J. Emery,J.L. Fourquet, "Topotactic $\mathrm{H}^{+} / \mathrm{Li}^{+}$ion exchange on $\mathrm{La}_{2 / 3-x} \mathrm{Li}_{3 \times} \mathrm{TiO}_{3}$ : New metastable perovskite phases $\mathrm{La}_{2 / 3 \times} \mathrm{TiO}_{3-3 x}(\mathrm{OH})_{3 \times}$ and $\mathrm{La}_{2 / 3 x} \mathrm{TiO}_{3 \times / 2}$ obtained by further dehydratation", Materials Research Bulletin, 33(11) 1681-1691 (1998)

11. Zview 2 for Windows (version 2.0), Scribner Assoc. Inc., Charlottesville, VA, USA, 2000. 